\title{
LA INTELIGENCIA PARA LA SEGURIDAD VECINAL DE ECUADOR: UN DESAFÍO EN TIEMPOS POSNORMALES
}

\author{
María Fernanda Noboa González* \\ Hernán Moreano Uriguen**
}

\section{Resumen}

El artículo es una reflexión crítica de la inteligencia como práctica compleja-ontoepistémica y metodológica-inscrita en el marco de la seguridad vecinal -frontera norte- de Ecuador, en tiempos posnormales. Actualmente, la comunidad de inteligencia ecuatoriana orienta su doctrina y práctica desde una perspectiva instrumental y politizada, con la creación de escenarios normativos, controlables y no caóticos. Sin embargo, los actuales tiempos posnormales, que determinan un futuro con múltiples flujos -inciertos, impredecibles, paradójicos y azarosos-, y concurrencia de disímiles actores, obligan a redimensionar su rol y naturaleza, con base en un replanteamiento, no solo del conocido "ciclo de inteligencia", sino de metodologías analíticas para identificar patrones de escenarios multidimensionales, articulados a las nuevas dinámicas del narcotráfico como factor de amenaza/oportunidad, para la ventaja decisoria en los diversos niveles de conducción del Estado. Los hallazgos muestran una emulación de las perspectivas ortodoxas de la inteligencia derivadas de los centros hegemónicos, alejadas de la realidad local.

Palabras clave: inteligencia crítica, posnormalidad, politización, metodologías adaptativas.

* Candidata doctoral en Estudios Internacionales de FLAcso-Ecuador (Ecuador). Máster en Gobernabilidad y Desarrollo de la Universidad Central del Ecuador. Decana del Centro de Prospectiva Estratégica del Instituto de Altos Estudios Nacionales (Ecuador) [maria.noboa.@iaen.edu.ec].

** Magíster en Relaciones Internacionales. Línea de investigación: Seguridad y geopolítica. Docente de la Escuela de Relaciones Internacionales en la Universidad Internacional del Ecuador (UIDE) (Ecuador). [hemoreanour@uide.edu.ec]. Recibido: 6 de marzo de 2018 / Modificado: 3 de julio de 2018 / Aceptado: 27 de julio de 2018.

Para citar este artículo

Noboa González, M. F. y Moreano Uriguen, H. (2018). La inteligencia para la seguridad vecinal de Ecuador: un desafío en tiempos posnormales. OPERA, 23, 97-114.

DOI: https://doi.org/10.18601/16578651.n23.07 


\section{INTELLIGENCE FOR ECUADOR'S BORDER SECURITY: A CHALLENGE IN POST-NORMAL TIMES}

\section{Abstract}

The article is a critical reflection of intelligence as a complex methodological and espistemological practice inscribed within the framework of the neighborhood security on northtern border of Ecuador in post-normal times. Currently, the Ecuadorian intelligence community guides its doctrine and practice from an instrumental and politicized perspective, with the creation of normative, controllable and non-chaotic scenarios. However, the current post-normal times, which determine a future with multiple uncertain, unpredictable, paradoxical and hazardous flows and the concurrence of dissimilar actors, force us to resize their role and nature, based on a rethinking, not only of the known " cycle of intelligence ", but of analytical methodologies. All this to identify patterns of multidimensional scenarios, articulated to the new dynamics of drug trafficking as threat / opportunity factor, for decision-making advantage in the various levels of State leadership. The findings show an emulation of the orthodox perspectives of intelligence derived from the hegemonic centers, far from the local reality.

Key words: Critical intelligence, postnormality, politicization, adaptive methodologies.
El pensamiento es tarea de vagos y maleantes. Hay que saber perderse para trazar un mapa: vagar por los márgenes y por el desierto, fuera de las fortalezas en las que están encerrados la verdad, el bien y la belleza. Solo los nómadas descubren otros mundos. Hay que saber pervertir la ley (jugar con ella) y a veces subvertirla (ponerla en cuestión) para cambiar y/o quitar la ley: provocar malos pensamientos en los bien-pensantes, asediar las sedes de la verdad, el bien y la belleza. Solo los malditos mejoran este mundo.

Jesús Ibáńez

\section{UNA ADVERTENCIA NECESARIA}

Disrupción, disidencia, descentramiento, desbordamiento de los márgenes del pensamiento único; repensar categorías y conceptos casi sacralizados en el campo de los Estudios de Inteligencia es el espacio de arenas movedizas ontoepistémicas y teórico-metodológicas en el cual se ubica este artículo. Se intenta redimensionar los "mantras positivistas" del mainstream de los estudios ortodoxos y heterodoxos ${ }^{1}$, los que focalizándose en examinar y evaluar casos en términos de sorpresas estratégicas o fallas de inteligencia, se han erigido en la columna vertebral del debate y la evolución del campo de inteligencia en "núcleos" duros - principalmente de Occidente-, de los que se derivan las voces de los otros, especialmente de los países de la región latinoamericana, que emulan los aparatos de reproducción intelectual (Tickner, 2013) que gravitan en un determinado

\footnotetext{
1 Arthur Onig (2007, p. 699) enfatiza que el marco teórico de la producción de la inteligencia es divergente en las dos principales escuelas: la Escuela ortodoxa argumenta que existen patologías y obstáculos inherentes a la tarea de inteligencia que hacen de cada atentado o ataque sorpresivo un éxito casi certero; mientras que la escuela heterodoxa afirma que las raíces de los ataques sorpresivos reposan en las inevitables fallas humanas de la gente de inteligencia.
} 
imaginario político y sentido del mundo, que no son necesariamente los prioritarios para nuestra región.

Con todo lo argumentado, el lector ha de tener en mente, que sibien el grueso de la producción de literatura académica se enmarca en los presupuestos teórico-metodológicos y analíticos de la literatura hegemónica-centrados principalmente en Estados Unidos, Canadá, Gran Bretaña y Países Bajos, que han desarrollado el contorno de líneas de investigación ${ }^{2}$-, muchos expertos consideran a los Estudios de Inteligencia como un área joven que se encuentra despuntando. Es más, según ellos, su campo de estudio responde a una particular dinámica que se encuentra en transición; en el denominado mundo poswestfaliano (Murillo, 2012; Sims, 2009). De su parte, otros autores consideran (Der Derian, 2008; Betts, 1988) que no se ha trabajado con suficiente dedicación para institucionalizar formalmente la literatura producida y la comprensión del campo de estudio, y la vinculación de este con sus prácticas en verdadero compromiso ético.

Con base en lo discutido, Stephen Marrin (2016) enfatiza, además, que una grave dificultad para dicha institucionalización es la escasa especialización de los estudiantes en esta área. Señala enfáticamente que "los estudios de inteligencia no han desarrollado una tradición académica, debido a que existen relativamente pocos trabajos doctorales en inteligencia” (p. 277). Este hecho se ha convertido en una seria dificultad para fortalecer los abordajes epistemológicos, que continúan siendo masculini- zados, centrados en el Estado, utilitaristas; en otras palabras, se han enfatizado los enfoques instrumentales. Es más, Marrin (2016), al ser uno de los autores seminales en la producción académica en inteligencia llega incluso a ironizar y sostiene que los que se dicen expertos en inteligencia, de cuando en cuando descubren la rueda conceptual, es decir, critica el trabajo de ellos desde el esquema de vino añejo en botellas nuevas.

A esta explicación es preciso sumar que varios expertos de la región latinoamericana, hace tres décadas aproximadamente, dieron prioridad a los Estudios de Inteligencia, con investigación y producción académica de gran relevancia (Swesson y Sancho, 2015; Maldonado, 2002; Cepik, 2001; Holzmann, 2004-2005; Ugarte, 2015; Gómez de la Torre, 2009). De manera tal que, en el marco de la pos-Guerra Fría, iniciaron reflexiones y debates coincidentes con las denominadas olas democratizadoras de la inteligencia estratégica, desde abordajes vinculados directamente con la consolidación de políticas públicas en la materia, de sus marcos legales y arquitecturas institucionales. Toda esta discusión y diseño de propuestas específicas se tejieron en torno a la conducción democrática de la inteligencia articulada a la gobernabilidad política del Estado. El propósito de este proceso fue establecer los contextos específicos y marcos de referencia pertinentes para institucionalizar sus prácticas en el avance de las democracias, considerando esencial el manejo y procesamiento de información en el establecimiento

2 Estas líneas no pueden considerarse aún, en sentido estricto, escuelas de pensamiento. 
de escenarios futuros, respecto de potenciales factores de riesgo, activación de amenazas a la seguridad o detección de oportunidades para el desarrollo del Estado.

De igual modo, se ha trabajado en torno a la consolidación de la cultura de inteligencia y el deseo de afinar nomenclaturas y lenguajes, con elementos de cohesión de las diversas comunidades por países, y sus dinámicas cooperativas en el ámbito internacional; concomitantemente se han debatido temas vinculados con el marco legal y las reformas legales que permitan su institucionalización.

Según lo dicho hasta este punto se puede resumir que, no obstante este grupo significativo de trabajos, es tarea aún pendiente la discusión del espacio definicional y teórico de los Estudios de Inteligencia que contemplen las particularidades de la región ${ }^{3}$ y sus necesidades, que sin marginalizar las nociones y los conceptos rectores del campo, es decir, sin intentar "descubrir el agua tibia”, incursionen en una propuesta crítica más allá de las narrativas fundacionales del mainstream. Dicha propuesta debería incluir una redimensión epistemológi$\mathrm{ca}$, que a su vez contemple el debate respecto de la responsabilidad ética en sus prácticas, y un compromiso político que garantice la gestión de la inteligencia estratégica orientada al bien común, es decir, no solo al cumplimiento de los objetivos de más alto nivel del Estado, sino a la responsabilidad ética frente a los derechos de los ciudadanos.
De igual modo, continúa inconclusa la discusión del lugar que les corresponde a los Estudios de Inteligencia en el entramado entre seguridad, estudios estratégicos, dimensión militar de la defensa -entre otros-, que también ponen en entredicho la función, el rol y la naturaleza de la inteligencia más allá del Estado, también tomando en cuenta que, como sostiene Alan DuPont (2005, p. 15), "se ha prestado poca atención a los cambios que se han dado en el manejo de la tecnología y la integración de los sistemas de inteligencia”, todo lo cual abre un sinnúmero de interrogantes respecto de la discusión en torno a los niveles entre la inteligencia estratégica y operacional, y la inteligencia y las operaciones propiamente dichas, lo que acarrea varias implicaciones, sobre todo en el relacionamiento entre los decisores políticos y los sistemas de inteligencia $y$, por ende, en la calidad de la toma de decisiones en diversos niveles

Estas y otras interrogantes aparecen asociadas también con la necesidad de contar con claridad definitoria y conceptual, habida cuenta de que estas prescripciones constituyen guías para la configuración de políticas públicas orientadas al logro de los objetivos nacionales y de la inserción internacional de los países latinoamericanos en los nuevos ambientes, caracterizados por la fluidez, lo caótico, lo cambiante, lo inesperado y la inestabilidad que plantea la globalización.

Al ser este un reto no menor, la presente propuesta sugiere redimensionar la concepción

3 La región es asimétrica, compleja, disruptiva, contradictoria, con diferentes prioridades, voluntades políticas, que se inscriben en las dinámicas transfronterizas, transregionales e intermésticas. 
ontoepistémica de la inteligencia como práctica compleja ${ }^{4}$ derivada e instituida desde un mundo turbulento -en palabras de Hastedt y Skelley (2009)-, en el que coexisten juegos asimétricos de poder y valores en disputa, y desde el cual no se busca solo la prevención de factores de riesgo y de la activación de las amenazas, sino la detección de oportunidades de alto valor estratégico para la maniobra de gobernabilidad de los Estados y el conjunto de la sociedad; con ello se sitúa a la inteligencia con un elevado valor agregado. Estas gravitan en medio de la dinámica de la arquitectura global y de los discursos y las nomenclaturas que subyacen a los nuevos entornos de la seguridad (nuevas amenazas, y comportamientos impredecibles de amenazas ya existentes), desde el entendido de que las prácticas del discurso en inteligencia -las denominadas metanarrativas $^{5}$ - también construyen sentidos de mundo respecto de la identidad de los Estados y otros actores no estatales, y reproducen y legitiman unas posturas frente a otras.

Por consiguiente, lo que se propone en definitiva es mirar la inteligencia críticamente, no tan solo como un subcampo de estudio de las relaciones internacionales, en un esquema fundacional y rígido, sino como un espacio de poder, todo lo cual aporta a la evolución del campo disciplinario y al avance de su teorización. Esta discusión, además, se inscribe en la reflexión en torno a una modalidad específica de producción de conocimiento, un saber que cobra el estatus de cognitivo experto de alto valor, producido por inteligencia. Este conocimiento, desde la mirada tradicional, sin duda incide en la forma de llevarlo a la práctica (toma de decisiones) sostenida en regímenes de verdad instituidos en dispositivos de discurso que legitiman sus acciones, en medio de disputas de poder entre sujetos y sus posiciones en la sociedad, como por ejemplo, entre los conductores estatales y los profesionales de los sistemas de inteligencia.

Con tal propósito se optó por develar, desde la perspectiva académica, los marcos conceptuales subyacentes a las prácticas profesionales que han priorizado abordajes pragmáticos y altamente utilitaristas de la inteligencia, que parecieran estar esculpidos en piedra y que, además, han marginalizado las consideraciones éticas para sus buenas prácticas. $\mathrm{Al}$ respecto, el propio experto Andrew Liaropoulos (2006, p. 6), del Departamento de Estudios Europeos e Internacionales de la Universidad de Piraeus (Grecia), incluso critica el hecho de que

\footnotetext{
4 Se refiere a la práctica compleja como una práctica multidimensional y dinámica, cuyo proceso-producto inscrito en una estructura institucional específica implica considerar su génesis, desarrollo y validación sin abstraerse del contexto sociohistórico de su producción, de los valores sociopolíticos y de los valores éticos (Rodríguez, 2010, p. 1) que la animan en medio de tensiones, heterogeneidad y discontinuidad entre los diversos actores involucrados (académicos, investigadores, profesionales, decisores). Tiene que ver además con prácticas que develan las dinámicas de poder y los dispositivos de discursos.

5 En los Estudios Críticos de Inteligencia, se entiende por metanarrativas o metarrelatos aquellas prácticas que construyen un sentido total, globalizador y universalizante, para explicar conocimientos y experiencias, que normalmente no son cuestionadas sino aceptadas como verdaderas, y que se alinean con las construcciones de discurso propias de la modernidad con características de esencialidad, fundacionalidad y binarismo.
} 
parte de la academia haya considerado a la inteligencia como un simple "género de estudio" y haya politizado su actividad, es decir, haya instrumentalizado la mirada que se tiene sobre ella. Es más, interpela duramente las explicaciones dadas en torno al $11 \mathrm{~S}$ por parte del Gobierno estadounidense respecto de lo ocurrido con la inteligencia nacional ${ }^{6}$, y también cuestiona el rol ontológico de la inteligencia en la "construcción de la hipótesis de la existencia de armas de destrucción masiva" erigida por el Gobierno norteamericano de Bush, que impulsó la invasión a Irak.

Lo dicho hasta aquí supone, entonces, que antes del $11 \mathrm{~S}$ no se había considerado la necesidad de tomar en cuenta a la revolución en asuntos de inteligencia, que se erigía tímidamente durante la pos-Guerra Fría. Luego del $11 \mathrm{~S}$ aparecía con claridad la necesidad de un cambio de rumbo, toda vez que la inteligencia estadounidense, considerada como una de las más potentes del mundo, se había visto interpelada por los ataques suicidas yihadistas de la red de $\mathrm{Al}$ Qaeda. Es más, a esto se sumaba la huella de vulnerabilidad en seguridad y moral que había dejado tal ataque. En consecuencia, se decidió la denominada reforma en inteligencia (consolidada desde el 2004), que buscaba un nuevo liderazgo mundial, y que exigía una adaptación radical en tanto institución, práctica, doctrina y cultura, a los nuevos ambientes de inteligencia que planteaba el mundo global sostenido en la inestabilidad estructural y la incertidumbre.
Para matizar lo dicho, de igual modo, hay que tomar en cuenta el desarrollo disciplinario como una contribución ineludible a la hora de evaluar las propias prácticas de inteligencia. En tal sentido, es pertinente la coincidencia de criterios de los expertos en la materia, Len Scott y Peter Jackson (2004, p. 2), quienes exigen situar la inteligencia como área académica, revisando las condiciones de su disciplinariedad y las posibilidades reales de su interdisciplinariedad, en tanto parte del estudio de las relaciones internacionales, transversalizada a su vez por la política y los estudios estratégicos/seguridad, entre otros. Con todo ello, la posibilidad de situar a la inteligencia como área académica va de la mano de la exigencia de abrir sus debates teórico-definicionales, por el momento como estudio de área de las relaciones internacionales, y, paulatinamente, como un campo autónomo. En este sentido, los expertos Andrew, Aldrich y Wark (2008) aseguran, entre otros argumentos, que tras el $11 \mathrm{~S}$ se puso sobre el tapete la aún débil sistematización académica de la inteligencia, desde miradas no ortodoxas ni revisionistas, y la limitada socialización de su naturaleza y rol en el debate público.

Con lo antes expresado, el lector deberá tener en mente que frente a dichas debilidades, este artículo se alinea, entonces, con una nueva perspectiva poco trabajada de la inteligencia. En definitiva, en términos de una práctica compleja ${ }^{7}$ constitutiva y constituyente de entornos caóticos, fluidos, azarosos, inciertos,

6 Las explicaciones dadas giran en torno a fallas y sorpresas estratégicas en la inteligencia, y no a una descoordinación con su conducción político-estratégica.

7 Aludimos no solo a la entrada fundacional de esta mirada del sociólogo y filósofo francés Edgar Morin, sino a la lectura situada del debate de la complejidad para el Sur, de los epistemológos Leonardo Rodríguez Zoya, Rolando García y Marcelo Manucci, entre otros. 
plagados de fenómenos emergentes, coincidentes con lo que varios investigadores han definido como tiempos posnormales (Funtowicz y Ravetz, 2000). Tales tiempos, incluso, han incidido en las propias concepciones de lo estratégico, uno de los factores determinantes en la doctrina de inteligencia, y vital en la configuración de un nuevo paradigma (Massoni, 2009 $)^{8}$. Dichos tiempos posnormales bien podrían homologarse con la concepción de los tiempos líquidos planteados por el filósofo polaco Zygmunt Bauman (2008). Practicar el arte de la vida, hacer de la propia vida una "obra de arte", equivale en nuestro mundo moderno líquido a permanecer en un estado de transformación permanente, a redefinirse perpetuamente transformándose (o al menos intentándolo) en alguien distinto del que se ha sido hasta ahora, por ende, la transformación del sentido y las implicaciones de la inteligencia son inevitables.

Estas consideraciones son imprescindibles para situar un nuevo locus de enunciación de la inteligencia como campo de estudio, disciplina y evolución teórica, revalorizando los aportes hechos desde el Sur'9. Recuperar las experiencias de los países sudamericanos implica, principalmente, realizar un ejercicio de sensibilidad cognitiva, responsabilidad ética y compromiso político que permita sacar a la luz no solo los presupuestos epistemológicos que subyacen a la escritura de las amenazas en la inteligencia, sino reivindicar su estudio pa- ra superar lo que el experto australiano James Der Derian (1992) define como el área menos teorizada de las relaciones internacionales, y que es preciso trascenderla del debate de la soberanía estatal, de la dimensión militar y su enfoque masculino. En este mismo sentido se considera, como sugiere Ken Booth (2007), incursionar teniendo en cuenta particularmente el alcance de los presupuestos teóricos y doctrinarios de la inteligencia, más que como simple teoría, como cuerpo del conocimiento en el nivel estratégico derivado de particulares perspectivas políticas, teóricas e históricas, que en suma se ven a sí mismas críticamente, y que son capaces de entender la necesidad de su propia evolución teórica-disciplinaria y pragmática, ante los permanentes desafíos de un mundo incierto, volátil y fluido.

Esto, como es obvio, incide en la escritura de las amenazas y la detección de oportunidades, como factor clave para la toma de decisiones y la conducción en los diversos niveles del Estado, empatados con imaginarios de seguridad y poder determinados (Campbell, 1992; Copeland, 2011). Es más, con esto se advierte que los imaginarios legitimadores del sentido de Estado y su identidad - por ende sus objetivos estratégicos-, frente a sí mismos y a los otros ${ }^{10}$, dejan de ser esencialistas y muestran que su debate está inserto en las mismas disputas por el poder. Esto alude también a que en el juego de las denominaciones y caracterizaciones de las amenazas -escritura, factores

\footnotetext{
8 “Nueva Teoría Estratégica: el paradigma emergente para la construcción y transformación de la realidad”.

9 No se pretende presentar como parte de un nuevo supermecado de escuelas de pensamiento (Booth, 2007, p. 29).

10 Se hace referencia a los otros Estados, otros actores, y a la dinámica en general del mundo internacional.
} 
de riesgo y oportunidades- existe algo más que un lenguaje simple, transparente y neutro. De hecho, detrás de los dispositivos de la escritura de las amenazas se dejan huellas de posiciones políticas específicas, derivadas de determinados intereses que influirán, como es obvio, en líneas de acción consonantes. Al respecto, es muy ilustrativa la reflexión del experto norteamericano David Campbell (1992), quien discute la influencia política en la definición de amenazas a la hora de construir agendas y políticas públicas en materia de seguridad.

Así, en el caso de Ecuador, sus perspectivas respecto de las dinámicas de la frontera norte han mutado y siguen mutando, como amenazas que están siendo conocidas con mayor preocupación, especialmente luego de la Firma del Acuerdo de Paz con el Perú (1998), momento en el cual el enfoque de la agenda de seguridad se centra en las fronteras con Colombia y en el impacto causado por el recrudecimiento del Plan Colombia (Gómez, 2002), particularmente, el punto de inflexión que implicó la Operación Fénix, en la zona de Angostura, ejecutada por las Fuerzas Militares colombianas el 1 de marzo de 2008, que detonó en el inicio del proceso de institucionalización del sector seguridad, incluida la configuración de una nueva arquitectura de inteligencia estratégica en el ámbito del Estado.

En este orden de ideas, las definiciones de las prioridades en seguridad están determinadas no solo por la orientación que el poder político ha dado a la configuración del "sentido y los efectos de las amenazas", sino a la caracterización que de ellas han construido los aparatos de inteligencia militar, policial, y desde 2009, la Secretaría Nacional de Inteligencia, como ente rector del Estado. En función de tales denominaciones, incluso se han diseñado programas y propuestas diversas: Unidad de Desarrollo del Norte (Udenor- 2001); Secretaría Técnica de Plan Ecuador (2007), y mecanismos tales como las comisiones binacionales de seguridad bifronterizas, para el monitoreo y la prevención de conflictos en las fronteras.

Con todo y eso, llama la atención que en los documentos rectores vigentes al 20142017 en materia de seguridad e inteligencia todavía no existe consenso respecto de la caracterización de las amenazas y de los sujetos vinculados a ellas, ni las potenciales situaciones críticas (conflictividad, tensiones, entre otras). No existen cartografías de riesgos y amenazas desarrolladas por ninguno de los órganos ejecutores del sector seguridad (Secretaría de Inteligencia, 2014; Ministerio Coordinador de Seguridad, 2014). El énfasis hecho es en denominaciones genéricas de grupos ilegales armados de Colombia ${ }^{11}$, sus líneas de acción y sus repercusiones, narcotráfico, secuestro, extorsión, crimen organizado trasnacional, entre otros.

De otra parte, los aportes conceptuales respecto de las dinámicas de fronteras, por parte de expertos internacionalistas y de seguridad, inciden en las diversas miradas a partir de las cuales son evaluadas las problemáticas 11 No hay una taxativa definición, porque bacrim, farcrim, gaos residuales, paramilitares, entre otros, funcionan
y actúan desde sus propias lógicas. 
gestadas en las fronteras con Colombia, y posicionan diversos puntos de vista respecto a las acciones que deberán tomarse frente a ellas (Cadena, 2011; Espinosa, 2008; Ramírez y Montúfar, 2007) y al consecuente y potencial diseño de políticas públicas.

En torno a la reflexión precedente, lejos de intentar cerrar la discusión, se deja abierta la postura respecto al "rol ontológico" de la inteligencia, en cualquiera de sus niveles, desde la "construcción" y definición de las amenazas; muchos expertos en la temática de seguridad, defensa y conflicto, como Frederick Massé (2004), David Campbell (1992) y Ken Booth (2007) ponen al descubierto la misma posibilidad de escribir las amenazas en tanto subjetividad perceptiva o instrumentalizarlas con fines ideológicos y políticos. Tal hecho, sin duda, contrariaría el marco teórico pertinente para comprender la configuración y condición local, transfronteriza e interméstica de las amenazas. Es más, se pone en grave riesgo el ejercicio y las prácticas de la profesionalización de la inteligencia, y se atenta contra su naturaleza emancipatoria, entendida como culturalmente sensible, pragmática, pero también universal por el aparecimiento de su politización.

Este punto es crucial porque ello incide en la construcción del sentido de los otros, en definitiva, de quién es amigo y quién enemigo (Der Derian, 2008), cuáles son los actores de riesgo y cuáles las oportunidades que los aparatos de inteligencia deben proactiva y prospectivamente plantear. En el caso ecuatoriano, se requiere una metodología compleja y sistémica que facilite situar de manera diferencial cada uno de los puntos de la frontera (fronteras) ${ }^{12}$, considerando también los mecanismos de evaluación de tendencias y escenarios multidimensionales y adaptativos a los nuevos desafíos, no solo globales, sino relacionados con las nuevas dinámicas y racionalidades gestadas por las estructuras macrocriminales colombianas, tras la firma del Acuerdo de Paz con las Fuerzas Armadas Revolucionarias de Colombia-Ejército del Pueblo (farc-er.)

\section{METODOLOGÍA CADUCA, VISIÓN BORROSA Y LIMITACIÓN EN LAS ACCIONES ESTRATÉGICAS}

Se ha hecho especial énfasis en la necesidad de repensar los aspectos metodológicos para comprender los fenómenos de seguridad e inteligencia relacionados con las realidades locales de nuestros países, amenazas y factores de riesgo, en la medida en que esto implica localizar el debate, es decir, sin dejar de ser globales, comprender en el marco de las coordenadas de la región y el país las problemáticas respecto de la inteligencia y su aplicabilidad. En tal sentido, luego de una discusión pormenorizada de las implicaciones ontoepistémicas y teórico-metodológicas de los campos de la seguridad e inteligencia en las realidades de los países de la región sudamericana, sus avances y limitaciones, se complementa este análisis con el caso concreto de Ecuador frente al conflicto colombiano, y las implicaciones que en

12 Los estudios tradicionales respecto de la frontera colombo-ecuatoriana la han situado de manera homogénea. 
diversos ámbitos este ha traído, y que ha sido motivo de producción de una vasta literatura de expertos nacionales y extranjeros (Torrijos, 2010; Bonilla, 2003).

Al ser el conflicto colombiano el que ocupa el quinto lugar entre los más complejos y críticos del mundo, cuyos costos han implicado graves pérdidas, a lo que se suman serios impactos de toda índole para las fronteras vecinales y la configuración de fuerzas y agudización de amenazas en la región, es pertinente considerar las implicaciones en el contexto geopolítico regional actual de la firma de paz entre el Gobierno colombiano de Juan Manuel Santos (2010-2018) y los grupos ilegales colombianos, especialmente con las FARC, toda vez que, según analistas como Jarrín (2017), Rivera (2016) y Vargas (2017), dicho conflicto ha dejado de constituir una amenaza para el Estado colombiano, y ha llegado a niveles de baja intensidad y concentración en las zonas de fronteras norte y sur con Ecuador. No obstante, otros autores sostienen que el acuerdo de paz no hace sino abrir el camino para la generación de diversos tipos de conflicto, no solo en la frontera interna de Colombia, sino que configurarán otra clase de tensiones, y evidencian la posibilidad de que se conformen nuevas amenazas mixtas, que pueden incidir en las dinámicas regionales, $y$, a partir de nuevas racionalidades macrocriminales, estas tensiones y conflictos podrían llegar a niveles insospechados (Echandía, Bechara y Cabrera, 2010; Niño, 2015; Duncan, 2015), lo que afectaría la gobernanza de las fronteras, la gobernabilidad política y el estado de la democracia en las relaciones bilaterales.
Dicho lo anterior, es lícito considerar que las herramientas de prospectiva, como complemento de la apreciación de inteligencia estratégica, presentadas a continuación, y que estaban alineadas a visiones europeas de futurología, pretendían descubrir los mecanismos que viabilizan los futuros posibles, $\mathrm{y}$ determinan que tan solo uno se convierta en el "más opcionado" en el tema de las negociaciones de Colombia. Es decir, que el alcance de esta prospectiva era absolutamente limitado y reducido a un escenario tendencial, cerrado y con una "supuesta" posibilidad de control de sus variables y los rangos de incertidumbre. La utilización de dicho conocimiento para que los presidente Santos y Correa lograran influir en el futuro y posteriormente analizaran las posibilidades de moldearlo, mejorando la calidad de las decisiones, resultaba restringida, y el análisis de dichas posibilidades mediante un mayor conocimiento de sus consecuencias también quedaba incompleto, sobre todo a la hora de evaluar las afectaciones de este proceso en el radio de acción vecinal, y en el caso concreto del Ecuador.

La apuesta de los organismos de seguridad de Colombia y Ecuador ha sido mantener el escenario de actividades ligadas al narcotráfico por parte de bandas delincuenciales que operarían en los departamentos de Narińo y Putumayo en Colombia, y en las provincias de Esmeraldas, Carchi y Sucumbíos en el Ecuador. El mapa 1 muestra las rutas del transporte de drogas en las fronteras norte y sur del Ecuador, y también a nivel marítimo.

Lo dicho hasta aquí supone que con los acuerdos de paz en Colombia se mantendrían 
MAPA 1. RUTAS DE TRANSPORTE Y ACOPIO DE DROGAS (ECUADOR)

\title{
Rutas de transporte y acopio de drogas
}

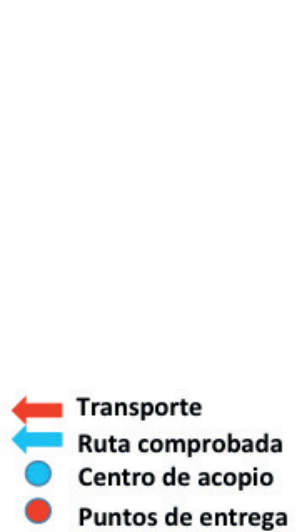

\begin{abstract}
Salida en lanchas rápidas y barcos pesqueros
\end{abstract}

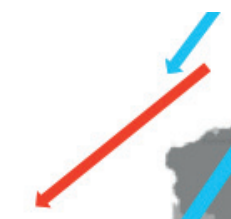

Buques mercantes

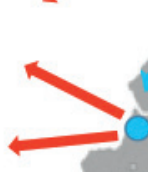

r.

Buques mercantes

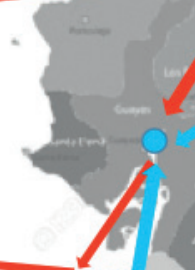

N

(x) 
escenarios multidimensionales que permitan planear mejores marcos de actuación, es decir, una gestión inteligente de las emergencias y los factores sorpresa. En suma, dirigir los esfuerzos a la comprensión de las amenazas, la capacidad de causar daño, sus nuevos mecanismos de evolución, entre otros, lo que quiere decir que la prospectiva, más allá de simples procedimientos metodológicos, impulsa a pensar con opciones diversas y en distintos niveles.

Lo dicho hasta aquí obliga a que los conductores estatales, los decisores de las entidades de seguridad y los profesionales de la inteligencia tengan la responsabilidad, no solo de afianzar una mirada estratégica para gestionar la incertidumbre, sino la voluntad política para el ejercicio de buenas prácticas prospectivas (Ortegón, 2007) útiles para la toma de decisiones en diversas circunstancias normales, disruptivas o cambiantes, lo que se traduce en un enriquecimiento tanto del marco cognitivo de los decisores como de sus habilidades y destrezas decisorias

Dicho de otra manera, la clave en la elaboración de inteligencia no está en la capacidad de descubrir información reservada u oculta, sino en la "capacidad de procesar, conectar y contextualizar la multitud de datos que ya están a disposición del analista de inteligencia" (Serra del Pino, 2014). De donde resulta la capacidad de lidiar con las contingencias, entendiendo inteligentemente las contradicciones y potenciales mecanismos de resolución. Esta dinámica alude a gestionar la inteligencia desafiando la mirada con la cual han sido percibidos los diversos fenómenos y acontecimientos, reconociendo la existencia de una inestabilidad estructural que obliga a planear nuevas maneras de pensar y actuar, a partir de estructuras flexibles y adaptativas (Manucci, 2010), para entender el contexto de los fenómenos y sus proyecciones en profundidad, esto es, comprendiendo la interacción entre variables, actores, contextos, marcos de referencia, alcances, entre otros.

En este mismo sentido, Jordi Serra del Pino (2014) indica que la información "está deviniendo progresivamente dinámica, por tanto, la interpretación de un dato en un momento o contexto dado no tiene por qué mantenerse indefinidamente o en otros contextos", lo cual implica que el trabajo de recolectores, procesadores, analistas también debe revisarse de manera constante. Esto es, no solo en tanto las acciones instrumentales, sino los puntos de enunciación de los productores de inteligencia, en una comprensión de la complejidad de los fenómenos y sus mutaciones. En un mundo fluido y líquido hay que tomar en cuenta que además de licuarse relaciones y vínculos, los entornos se tornan más vulnerables. Por tal motivo, y con base en una perspectiva crítica de la inteligencia estratégica, se reconoce, entonces, que cada profesional se irá transformando conforme se transforma el objeto de estudio, a partir de una disposición política, compromiso y responsabilidad ética frente a la producción de conocimiento experto para la toma de decisiones en los diversos niveles de la conducción del Estado (Rodríguez, 2010).

Para matizar la lógica argumental, en el mapa 2, y con base en datos del sistema de inteligencia policial ecuatoriano, se puede evidenciar la presencia de redes de tráfico de personas que operarían en Perú, Ecuador y Colombia con fines de explotación sexual. Estas estructuras 
MAPA 2. TRATA CON FINES DE EXPLOTACIÓN SEXUAL

\section{Trata con fines de explotación sexual}

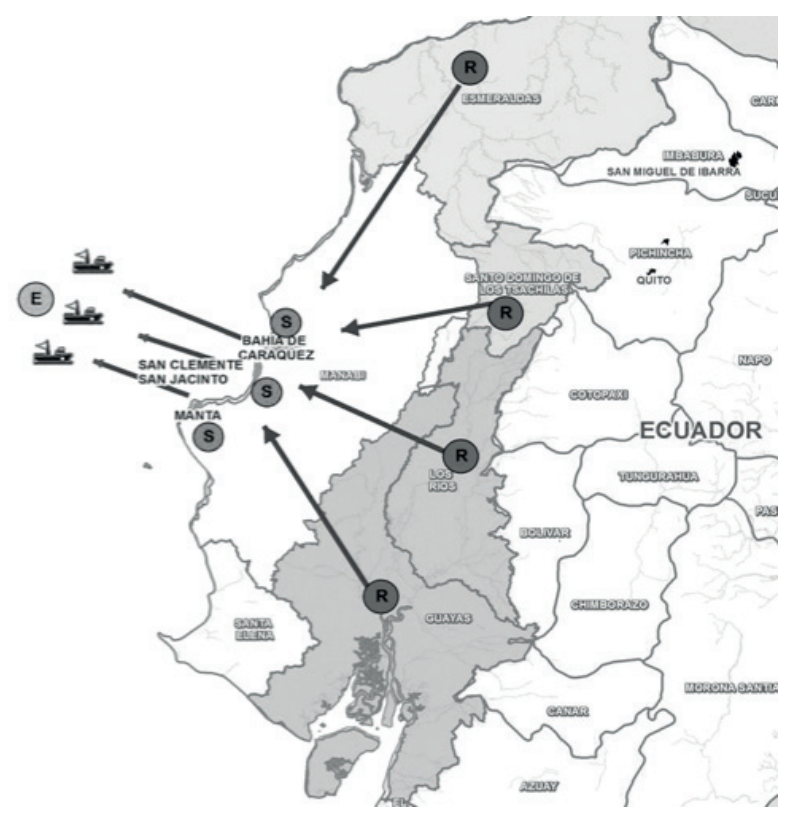

estarían compuestas por las mismas bandas de narcotraficantes que le "apuestan a otros negocios ilícitos" (Carrión, 2012).

Con base en ello, y precisamente por la enormidad y diversidad de territorios que deben ser cubiertos para entender los múltiples factores de riesgo y amenazas a la seguridad, y también las oportunidades, es que deben redimensionarse las acciones de inteligencia. Así, el experto espańol Jordi Serra (2014) llega a proponer la creación de redes de confianza como mecanismo que permita incluir a otro tipo de actores, más allá del sistema de inteligencia, para generar una información validada, de confianza, que pueda ser utilizada por la comunidad de inteligencia. La idea de crear una comunidad ampliada de inteligencia con elementos externos (academia, movimientos sociales, líderes de opinión, líderes gremiales, tanques pensantes, entre otros) no debe ser una propuesta nueva. Esta, sin duda, no es una idea descabellada si se quiere institucionalizar la comunidad de inteligencia en Ecuador.

La Secretaría de Inteligencia, como el ente rector de la inteligencia del más alto nivel en Ecuador, tiene a su cargo procesar toda información, con nuevas metodologías de análisis, con el fin de generar inteligencia estratégica y detectar amenazas, vulnerabilidades y factores de riesgo en el territorio, tomando en cuenta siempre que tales metodologías puedan ser adaptadas a las propias condiciones locales (Holzmann, 2004-2005). Por ello, la relación inteligencia-análisis, sin duda instrumentalizada desde perspectivas teóricas racionalistas de los estudios de relaciones internacionales, 
y de los estudios estratégicos de seguridad e inteligencia "debe ser redimensionada para interpretar cabalmente un mundo internacional turbulento, incierto, azaroso" (Serra del Pino, 2014). Y es precisamente la mirada crítica la razón de ser de este estudio, así como los desafíos que tienen los países de la región dada su morfología asimétrica, sus crisis de gobernabilidad, la coexistencia de factores adversos, las rupturas, las heterogeneidades sociales, políticas, entre otras, que no admiten perspectivas lineales para su comprensión, que resultan limitadas y reduccionistas.

En suma, como se ha insistido, es imprescindible rebasar la mirada estática sobre prácticas instrumentales de inteligencia y adentrarse en las nuevas aproximaciones teóricoconceptuales dinámicas, que den cuenta de las necesarias transformaciones de las comunidades de inteligencia y los principios doctrinales que guían su accionar (Hastedt y Skelley, 2009, p. 122), para evitar caer en una mirada esencialista o en la creencia de que el mundo todavía funciona como una máquina sobre la cual se puede tener control (Manucci, 2010).

Tales prácticas instrumentales se sostienen en los mitos de las relaciones internacionales, a decir de Chyntia Weber (2010, p. 218), un proceso a partir del cual, al parecer, las metodologías de la inteligencia se han naturalizado como

... prácticas homogéneas y dominantes de la naturaleza objetiva del mundo internacional y sus factores de riesgo y amenaza -veraces-, a partir de un claro silenciamiento de múltiples voces y poca comprensión de los intersticios y contextos azarosos, heterogéneos contradictorios e híbridos de la política mundial (2010, p. 218).

\section{CONCLUSIONES}

La comunidad de inteligencia en el Ecuador debe reconocer la necesidad de ampliar y diversificar, no solo las fuentes de inteligencia, sino también los métodos, enfoques, perspectivas y teorías. Es necesario trabajar con nuevas metodologías que permitan crear el mayor número de escenarios y no apostarle a un solo método.

El análisis posnormal revela que el reto es mucho mayor que una mera adaptación metodológica a los nuevos tiempos, se trata de un desafío epistemológico para el que aún no se está preparado; por ende, es inexorable el aporte de la academia para este desafío cognitivo.

Se tiene el profundo convencimiento de que si se definiera la inteligencia posnormal como aquella que se acciona cuando los datos son inciertos, los valores están en disputa, los riesgos son elevados y las decisiones por tomar son urgentes, la respuesta mayoritaria de los servicios de inteligencia sería que eso no es posnormal, sino lo "normal". Es preciso renovar las metodologías de análisis y la mirada respecto de las nuevas rutas y modelos analíticos. Urge trabajar con un "conjunto de especialistas" que colaboren con los servicios de inteligencia, sin ser miembros de ellos, en la producción de inteligencia. Esta práctica permitiría un mayor enriquecimiento de información para el análisis, pues se tendría a la vez una visión más especializada en contextos transdisciplinarios.

En el caso de la definición de amenazas y factores de riego en la frontera de Ecuador y Colombia es necesario crear una comunidad epistémica que ayude a la creación de escenarios 
y análisis prospectivos, que vayan más allá de la identificacón de actores, redes y estructuras. Es necesario trabajar en escenarios mutables $\mathrm{e}$ inciertos en la seguridad global.

Ante lo discutido surgen muchas inquietudes, sobre todo con respecto a una propuesta alternativa teórica de lectura de las relaciones internacionales y seguridad global, habida cuenta de que el mundo empírico no está divorciado del mundo teórico, y aquellos dos mundos tampoco lo están de los procesos de las metodologías de investigación y análisis, que son múltiples, abiertos y que se encuentran enfrentados a la indeterminación, al azar, a los sucesos emergentes, y en los cuales los actores del mundo internacional se ven desafiados en su marcos cognitivos y éticos, al igual que los académicos.

En este mismo orden de ideas, hay que reconocer que la propia ontología de las relaciones internacionales y la seguridad ha mutado considerablemente y, consecuentemente, la naturaleza del poder, lo que exige desde el ámbito académico un pensamiento estratégico-prospectivo, con nuevas ópticas que permitan complejizar su lectura, incorporando múltiples voces, el cambio, la discontinuidad y la ruptura. Esto evitará la esencialización de los fenómenos y abrirá múltiples posibilidades para evaluar los fenómenos producto de la inteligencia y el análisis prospectivo dentro de espacios inter y transdisciplinarios, que interpelan problemas lógicos, metodológicos y del lenguaje en los campos de estudio tales como las relaciones internacionales, los estudios críticos de inteligencia y la seguridad global.

Por último, es necesario que la Comunidad de Inteligencia brinde una definición de "cultura de inteligencia" como un conjunto de conocimiento que la sociedad civil debe tener sobre la finalidad, el rol y el requerimiento de un servicio de inteligencia, de manera que perciba como suyas las cuestiones relacionadas con seguridad, libertad y la defensa de sus intereses en el marco de democracias maduras y participativas.

\section{REFERENCIAS}

Andrew, C., Aldrich R. y Wark., W. (2008). Secret Intelligence. A Reader Theory of world security. Cambridge: Cambridge University Press.

Bauman, Z. (2008). Modernidad líquida. México: Fondo de Cultura Económica.

Bonilla, A. (2003). El conflicto colombiano y su impacto en los paises andinos. Bogotá: Universidad de los Andes, CEso.

Booth, K. (2007). Theory of World Security. Cambridge: Cambridge University Press.

Cadena, J. (2011). Plan Colombia y dinámicas de seguridad Ecuador- Colombia. Comentario Internacional, 1, 121-149. Quito. Universidad Andina Simón Bolívar. Recuperado de http://repositorio. uasb.edu.ec/bitstream/10644/3445/1/07-TCCadena.pdf

Campbell, D. (1992). Writting Security. United Statess Foreing Policy and the Politics of identity. Minneapolis: Minnesota Press.

Carrión, F. (2012). El Ecuador un Hub del Narcotráfico. Revista Vistazo, julio.

Cepik, M. (2001). Segurança Nacional e Controle Público: Limites dos Mecanismos Institucionais Existentes. Contexto Internacional, 23, Issue 2. 
Chávez Cepik, M. (2001). Servicios de Inteligencia. Agilidad y transparencia como dilemas de institucionalización (Tesis de Doctorado en Ciencia Politica ). IUperj Instituto Universitário de Pesquisas do Rio de Janeiro. Brasil

Copeland, L. (2011). Make Intelligence, Not War. Reframing Security with Antidiplomacy. En L. Crowe, New Directions, The future of Canadian (In) Security Studies (pp. 37-48). York: York University.

Der Derian, J. (1992). Antidiplomacy. Spies, Terror, Speed and War. Estados Unidos: Wiley- Black-Well.

Der Derian, J. (2008) Antidiplomacy, Intelligence Theory and Surveillance. Intelligence and National Security, 8 (3), 29-51. https://www.tandfonline.com/doi/abs/10.1080/02684529308432213

Duncan, G. (2015). Exclusión, insurrección y crimen. En Contribución del entendimiento del conflicto armado en Colombia. Bogotá: Comisión Histórica del Conflicto y sus Víctimas. Recuperado de www.scielo.org.co/scielo.php?script=sci_nl inks\&ref=000151\&pid=S0486-6525201500 010001000032\&lng=pt

DuPont, A. (2005). Intelligence for the twenty -first century. En K. Wesley. Twenty First Century Intelligence (pp. 14-39). London: Routledge.

Echandía, C., Bechara, E. y Cabrera, I. (2010). Colombia: estado del conflicto armado al final de la administración de Álvaro Uribe. En H. Mathieu y C. Niño (eds.). Anuario 2010. (pp. 136-172). Bogotá: Friedrich Ebert Stiftung - Programa de Cooperación en Seguridad Regional.

Espinosa, R. (2008). Las fronteras con Colombia. Quito: Corporación Editora Nacional. Universidad Andina Simón Bolívar. http://www.uasb.edu. ec/publicacion?las-fronteras-con-colombia- 400
Funtowicz, S. y Ravetz, J. (2000). La ciencia posnormal. Barcelona: Icaria.

Gómez de la Torre, A. (2009). Servicios de Inteligencia y democracia en América del Sur. ¿¿Hacia una nueva generación de reformas normativas? Agenda Internacional, (3), 119-130. Recuperado de https://dialnet.unirioja.es/descarga/articulo/6302509.pdf

Gómez, V. (2002). Reflexiones de la seguridad nacional y política exterior. Quito: Flacso.

Hastedt, G. y Skelley, D. (2009). Intelligence in a turbulent world: Insights from organization theory. En P. Gill, S. Marrin y M. Phythian. Intelligence Theory: Key Questions and Debates. London: Routledge.

Holzmann, G. (2004-2005). La inteligencia estratégica en países emergentes. Chile: Instituto de Asuntos Públicos Universidad de Chile. Recuperado de http://www.agendapublica.uchile.cl/n5/2_holzmann.html

Jarrín, O. (2017). Seguridad complementaria. Diario El Comercio, noviembre

Liaropoulos, A. (2006). A (r)evolution intelligence affairs? In search of a new paradigm. Research Institute for European and American Studies, 1-19.

Maldonado Prieto, C. (2002). Servicios de Inteligencia en Sudamérica. Estado de Situación en una perspectiva comparada. Georgia: Instituto para la Cooperación de seguridad hemisférica. Recuperado de www.scielo.br/scielo.php?script=sci_nli nks\&ref=000141\&pid=S0104-8775200700020 $001000027 \& \operatorname{lng}=$ en

Manucci, M. (2010). Contingencias. 5 desafios de cambio para una nueva década. Bogotá: Norma.

Marrin, S. (2016) "Improving Intelligence Studies as an Academic Discipline". Intelligence and National Security Journal. 
Massé, F. (2004). Las dimensiones regionales: el conflicto colombiano entre la exageración e instrumentalización de la amenaza. En Encrucijadas de la seguridad en Europa y las Américas (pp. 395-408). Bogotá: (CEPI)/Centro Editorial, Universidad del Rosario.

Massoni, P. A. (2009). Hacia una teoría general de la estrategia. El cambio del paradigma en el comportamiento humano, la sociedad y las instituciones. Barcelona: Ariel Editores.

Ministerio Coordinador de Seguridad (2014). Plan Nacional de Seguridad Integral. Quito: Ministerio Coordinador de Seguridad. Recuperado de http://instrumentosplanificacion.senplades.gob. ec/documents/20182/22941/PlanNacionaldeSeguridadIntegral2014-2017.pdf/f60ca2ad-41d64c1b-9b0d-05336e548f5f

Murillo, C. (2012). Diplomacia, inteligencia exterior y secreto diplomático frente a los desafíos de la era global. Revista Policía y Seguridad Pública, 5 (2).

Niño, C. (2015). Cerca de un acuerdo?... Cerca de la paz? El laberinto de las negociaciones en Colombia. Nueva Sociedad. Democracia y politica en América Latina. Buenos Aires: Friedrich Ebert Stiftung. Recuperado de http://nuso.org/autor/ catalina-nino/

Onig, A. (2007). A new direction for theory-building in intelligence studies. International Journal of Intelligence and CounterIntelligence, 20, 699716. doi:Dor: 10.1080/08850600701249840

Organización de Naciones Unidas (2010). Declaración de Palermo. unODC.

Ortegón, E. (2007). Manual de prospectiva y decisión estratégica: bases teóricas e instrumentos para América Latina y el Caribe. Recuperado de https:// books.google.com.ec/books?isbn $=9213228848$
Ramírez, S. y Montúfar, C. (2007). Colombia-Ecuador. Cercanos y distantes. Bogotá: Editorial Unilibros.

Rivera, F. (2016). Fredy Rivera plantea siete medidas para reformar la lucha contra el narcotráfico. Diario El Comercio, enero.

Rodríguez Zoya, L. (2010). Complejidad de la relación entre ciencia y valores. La significación política del conocimiento científico. Documento de jóvenes investigadores. Buenos Aires: Instituto de Investigaciones Gino Germani, Facultad de Ciencias Sociales, Universidad de Buenos Aires.

Scott, L. y Jackson, P. (2004). The study of intelligence in Theory and practice. Intelligence and National Security, 19(2), 139-169. doi:Dor: 10.1080/0268452042000302930

Secretaría Nacional de Inteligencia (2014). Agenda Sectorial de Inteligencia. Quito: Secretaría Nacional de Inteligencia. Recuperado de http:// instrumentosplanificacion.senplades.gob.ec/ documents/20182/22941/AgendaSectorialdeInteligencia2014-2017.pdf/5f001e6f-ee99-4a04$887 \mathrm{f}-55 \mathrm{da} 66 \mathrm{e} 03313$

Serra del Pino, J. (2014). Los retos de la inteligencia en tiempos posnormales. Barcelona: Universidad de Barcelona.

Sims, J. (2009). Defending adaptative realism. Intelligence theory comes of age. En P. S. Gill. Intelligence Theory. Key Questions and Debates (pp. 151-165). New York: Routledge.

Swesson, R. y Sancho, C. (2015). Gestión de la Inteligencia en las Américas. Defending adaptative realism. Intelligence theory comes of age. Washington: Ni Press.

Tickner, A. (2013). Core, periphery and (neo) imperialist IR. European Journal of International Relations, 3. 
Torrijos, V. (2010). Crisis, paz y conflictos. Bogotá: CEPI - Universidad del Rosario.

Ugarte, J. M. (2015). El ámbito normativo de la inteligencia interior en América Latina. Varia Historia, 28, (47), 121-162. Recuperado de www.scielo. br/pdf/vh/v28n47/07.pdf
Vargas, A. (2017). Reflexiones y perspectivas del proceso de paz en Colombia. Revista Semana, abril. Weber, C. (2010). Interruption Ashley. Review of International Studies, 36 (4). 